\title{
A Partitioned cab system based on population and destination
}

\author{
Liming Liu \\ School of Electrical Engineering, North China Electric Power University, Baoding 071003, China \\ 839228791@qq.com
}

Keywords: cab system, average waiting time, no-load ratio.

\begin{abstract}
The degree of satisfaction of the customers lies largely in two major factors: the average time of waiting a cab and the total cost of boarding a cab. We create a basic model which has two parts: to calculate the total number of the cabs that can limit the average waiting time, and to optimize the prices that can satisfied both the customers and the companies. The major assumption is that we divide the research area into 9 parts, with 9 centers in them. Our target customers are those who relying on cabs and assume that everyone is going to the airport. We first calculate and solve the total number of the cabs in the area based on the limit of the average waiting time. We define a ratio named no-load ratio which is related to the density of population. Finally we compare analytically and numerical results with reality and validate that our method is correct and robust.
\end{abstract}

\section{Introduction}

Mythical is a vibrant college town in the middle of Dompkins County (upstate NY). Local landmarks \& destinations include parks, gorges and waterfalls, a lake, downtown with shops, restaurants \& theaters, a large university, a smaller liberal arts college, a shopping mall, and the Mythical-Dompkins Regional Airport. The geographic layout is such that most locals own and use cars on a daily basis. Still, a significant portion of the population relies on public transportation and taxicabs. This is particularly true for those with lower incomes and temporary residents (e.g., the students). All others use taxis infrequently -- primarily when traveling to/from the airport or the bus station. Unfortunately, many customers are unhappy with both the quality and the price of services offered by the local taxi companies. Numerous complaints can be found on Yelp and in other online forums.

Table 1 Model Parameters

\begin{tabular}{cc}
\hline Parameter & Meaning \\
\hline$N$ & The number of the taxicabs \\
$N_{\text {working }}$ & The volume of services of the cab \\
$S$ & The total mileage of cabs \\
$\delta$ & proportion of flexibility \\
$p$ & the number of the population \\
$\mu$ & the rate of the target customer \\
$a_{i}$ & the percentage of total population in the part \\
$G$ & the average number of customers in one cab \\
$G$ & volume of transport(times of the boarding) \\
$g$ & the percentage of travelling by taxi \\
$\bar{T}$ & the average serving time \\
$\bar{D}$ & the average of the velocities \\
$S$ & the average of the distance per carriage \\
$t$ & the total mileage \\
$q$ & the waiting time of the customers \\
$p_{(x, y)}$ & no-load ratio \\
& the density of population at the certain point $(x, y)$ \\
\hline
\end{tabular}


Based on the problem ,we need to solve :what is the total number of cabs needed to serve the "Greater Mythical" area. The goal is to ensure that a) the average customer waiting time is $\leq 15$ minutes; b) less than $10 \%$ of customers end up waiting more than 25 minutes.

For the first, we concentrate on the different parts and the average waiting time of customers. The parameters we assume and use are shown in Table 1.

\section{Number of taxicabs}

Step 1 No-load ratio $(q)$ First we study the statistics from the of official website [4], which shows the density of population among different areas. To image-building the relationship, we plot the Figure 1. As the Figure 1 shows that different parts have different density of population, which relies on the destinations and landmarks. $(x, y)$ is coordinate variable of areas, especially for 9 centers, while $\mathrm{z}$ is the density parameters.

We generally assume that the no-load ratio accord to the expression.

$$
q \propto \frac{\rho_{(x, y)}}{\rho_{\max }}
$$

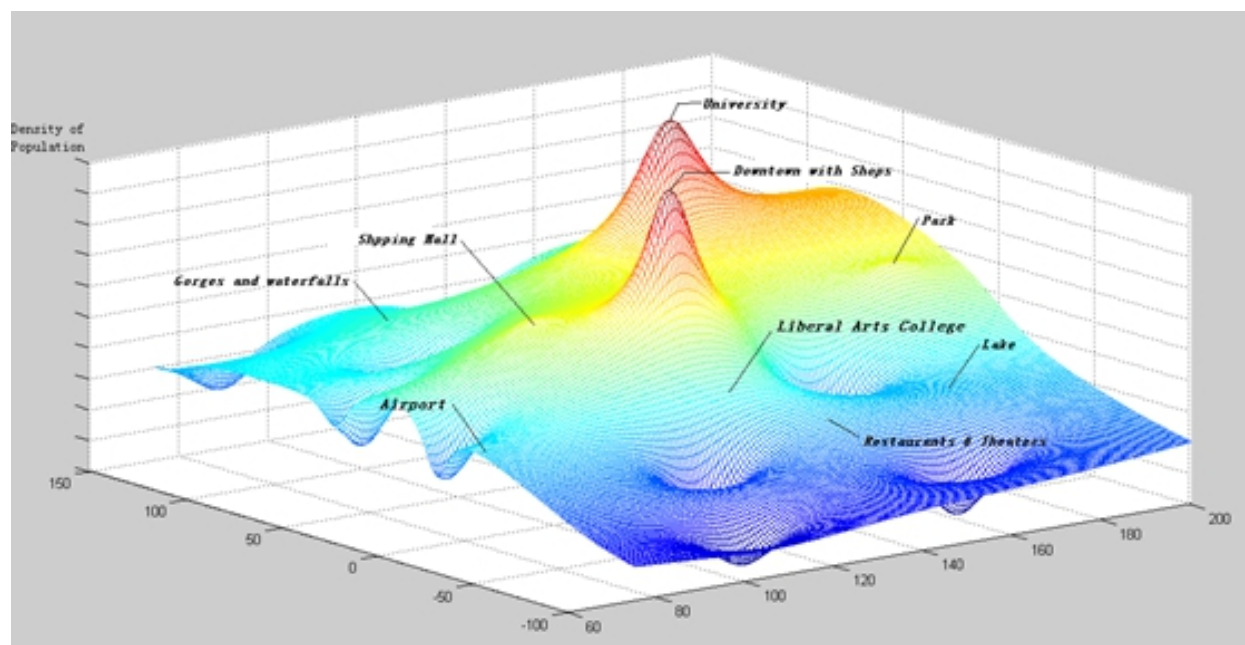

Fig. 1 The density of population

Step 2 volume of mileage $(S)$

To find out the volume of mileage, we should first find out the volume of transport. We assume that the number of the population is $P$, of which the percentage of travelling by taxi is $k . h$ means the average number of customers in one cab.

We have

$$
\boldsymbol{G}=\frac{\boldsymbol{p} \boldsymbol{g}}{\boldsymbol{h}}
$$

We know it from the problem statement that others use taxis infrequently, primarily when traveling to/from the airport or the bus station. So we isolate it as the driving factors.

$G a_{9}$ is the total times of traveling to the airport. The total distance is ` $D G a_{9}$.

We have the expression for $S$.

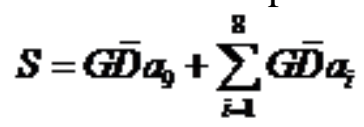

Step 3 volume of service ( $\left.N_{\text {working }}\right)$.

$N_{\text {working }}$ is a parameter which is determined by the total number of the taxicabs $(N)$ and the proportion of flexibility $(\delta)$. This relationship is intuitive.

Thus, $N$ can be represented as 


$$
\boldsymbol{N}=\frac{\boldsymbol{N}_{\text {morkis }}}{\boldsymbol{\delta}}
$$

The total mileage of the cab is $S$. While the number of the cab boarding passengers is $N_{\text {working. }}$ We have an equation for $q$ :

$$
q=1-\frac{S}{\bar{T} \bar{W} N_{\text {watie }}}
$$

The result of investigations in some cities shows that traffic reaches its peak from 8:00 am to 8:00 pm, which may include $90 \%$ of the volume of travel [5], there are differences in the source of the passengers between day and night. Therefore, the number of the cabs is based on the daytime. Then we have the equation for $N_{\text {working }}$ :

$$
\text { Noorting }=\frac{09 S}{\bar{T} \bar{V}(1-q)}
$$

From the equations above, the relationship between q and $N_{\text {working }}$ is acquired. Now we can pay our attention to the waiting time. We move to step 4.

Step 4 waiting time $(t)$ A model is created for waiting time. That may be explained by the fact that the waiting time has the inverse relationship with the no-load ratio, but not only the no-load ratio. Since the relationship is complicated, we put in a parameter as $c_{2}$. We assume $\mathrm{k}$ as a scale coefficient to find the inverse proportion. From the plot 2, we are able to conservatively conclude that it meets the equation. Because the no-load ratio is nearly impossible to become zero, we add a parameter " $\mathrm{C}_{1}$ " to estimate.

Then we have

$$
\boldsymbol{t}=\frac{\boldsymbol{k}}{\left(c_{1}+q\right)^{\mathbf{a}}}+c_{2}
$$

To find the average time, we have the definite integral

$$
\bar{t}=\int_{0}^{1} t d q
$$

From the 4 expressions, we find the relationship between $\mathrm{t}$ and $\mathrm{N}$. That is

$$
N=\frac{09 S}{\bar{T} \bar{V}\left(1+c_{1}-\sqrt{\frac{k}{t-c_{2}}}\right)}
$$

\section{Summary}

Our goal is to ensure that the average waiting time is less than 15 minutes while less than or equal to $10 \%$ of the customers would be waiting at least 25 minutes.

The waiting time is determined by the no-load ratio, total mileage, the average of the serving time and velocities. Since the standard of the parameters have already been calculated, we can use our model to evaluate the total number. We set to work accumulating a mass of data. As the population is 8170,000 , the rich is 390,000 and $\mu$ is generally 0.95 . We find the g to be $3 \% . S$

The value of $\delta$ is 0.86 . We assume the population to be in 140,000 as it mentioned in introduction. $h=1.7, a_{9}=5 \%, \bar{D}=5.18(\mathrm{~km}) \quad \bar{T}=13.25(\mathrm{~h}) \quad \bar{V}=32(\mathrm{~km} / \mathrm{h})$

Then we calculate the $G$ to be 2470 times. The total mileage (S) is $24341 \mathrm{~km}$ [6]. For the average $\overline{\mathrm{t}}$ $=15 \mathrm{~min}$, we validate $\mathrm{q}$ to be 0.4 through the expressions. The consequence is that

$N_{\text {working }}=86$

$N=100$

We validate that our results agree with practice and are robust and correct [9]. 


\section{References}

[1] Levy, Francesca. America's Best Places to Raise a Family. Forbes.com. June 7, 2013

[2]Yimeng Guo, the service of the taxi in Manhattan, NY. The People Net. http:// auto. people. com. cn/n/2015/0119/c1005-26407605. html 1.19.2015

[3] S, Xin. J, Lin and Y, Xiao. 2005 the integrated model of City taxi price planning NPMCM

[4] Information on: http://www.cityofithaca.org/391/Information-for-Residents

[5] Q, Sun, Y Xiang and X, Liu 2011. Queueing theory of predicting the optimal number of taxi CUMCM

[6] Hao, Ding, 2008 Planning model of cabs in city management. Mathematics in practice and theory. Vol38. N.6 March 2008

[7] Information on: http://wk.baidu.com/view/

[8] Bairan Zhang, "Chinese Dictionary" encyclopedic knowledge of Nanjing University press, 1992

[9] Information on: http://www.zuzuche.com/taxi/taxi_city.php?city=785 